\title{
Association of retinol binding protein-4, cystatin $C$, homocysteine and high-sensitivity C-reactive protein levels in patients with newly diagnosed type 2 diabetes mellitus
}

Yun-Sheng Wang ${ }^{1,2}$, Jun Ye², Xiao Yang ${ }^{3}$, Gui-Ping Zhang ${ }^{3}$, Yong-Hong Cao², Rong Zhang ${ }^{2}$, Wu Dai², Qiu Zhang ${ }^{1}$

1Department of Endocrinology, the First Affiliated Hospital of Anhui Medical
University, Hefei, Anhui, China
${ }^{2}$ Department of Endocrinology, the Second Hospital of Hefei City, Hefei, Anhui, China
${ }^{3}$ Department of Ultrasonography, the Second Hospital of Hefei City, Hefei, Anhui, China

Submitted: 5 August 2018

Accepted: 30 September 2018

Arch Med Sci 2019; 15 (5): 1203-1216

DOI: https://doi.org/10.5114/aoms.2018.79565

Copyright (c) 2018 Termedia \& Banach

\section{Abstract}

Introduction: To investigate the serum retinol binding protein (RBP)-4, cystatin C (Cys C), homocysteine (HCY) and high-sensitivity C-reactive protein (hs-CRP) levels in newly diagnosed type 2 diabetes mellitus (NT2DM) patients, prediabetes mellitus (PDM) subjects and normal controls, as well as their correlation with clinical and laboratory indexes, such as blood pressure and lipoprotein.

Material and methods: A total of 242 subjects, including 141 NT2DM patients, 48 PDM subjects and 53 healthy controls, were recruited in the present study. Serum RBP-4, Cys C and hs-CRP concentrations were measured by enzyme-linked immunosorbent assay (ELISA). HCY concentration was determined by the chemical luminescence method.

Results: There were significant differences in Cys C and hs-CRP among NT2DM patients, PDM subjects and normal controls. In comparison to controls, there were significantly elevated Cys $C$ and hs-CRP levels in PDM (both $p<0.001)$, and a significantly increased Cys C level in NT2DM $(p<0.001)$; however, there were no significant differences in Cys $C$ and hs-CRP levels between NT2DM and PDM, and no significant differences of hs-CRP levels between NT2DM and normal controls. No significant differences of RBP-4 and HCY levels among NT2DM, PDM and normal control groups were observed. Conclusions: Aberrant Cys $C$ expression and its clinical associations in NT2DM suggest their important role in this disease.

Key words: retinol binding protein-4, cystatin C, homocysteine, highsensitivity C-reactive protein, type 2 diabetes mellitus.

\section{Introduction}

Type 2 diabetes mellitus (T2DM) is one of the most common chronic metabolic diseases, featuring hyperglycemia resulting from resistance to insulin action and an inadequate compensatory insulin secretory response [1]. The chronic hyperglycemia of diabetes is associated with long-term damage, dysfunction and failure of various organs, of which the long-term complications, such as retinopathy, nephropathy, cardiovascular (CV) and cerebrovascular diseases, are considered to be the

\author{
Corresponding authors: \\ Qiu Zhang \\ Department of \\ Endocrinology \\ the First Affiliated \\ Hospital of Anhui \\ Medical University \\ Hefei 230022 \\ Anhui, China \\ Phone: +8655162922069 \\ Fax: +8655162922069 \\ E-mail: zhangqiu1965@sina. \\ com
}

Wu Dai

Department of Endocrinology the Second Hospital

of Hefei City

Hefei, Anhui

230011, China

Phone: +8655162965336

Fax: +8655162965336

E-mail: daiwu1984@sina.com 
dominant factors affecting the life span and the quality of life in T2DM [2-5].

Cystatin C (Cys C), a cysteine proteinase inhibitor, is a non-glycosylated single chain protein with a molecular weight of $1.3 \mathrm{kDa}$. It is secreted shortly after its synthesis and steadily produced by all nucleated cells $[6,7]$. Investigations have demonstrated that serum Cys $C$ improves estimates of glomerular filtration rate (GFR) more than serum creatinine $(\mathrm{Cr})$-based methods alone, and was associated with the presence of $\mathrm{CV}$ disease in subjects with mild renal impairment [8-10]. It has been reported that Cys $C$ levels increase and cysGFR levels decrease with increasing severity of glucose intolerance [11]. Moreover, in patients with diabetes, several studies have demonstrated that serum Cys $C$ had better performance compared with serum $\mathrm{Cr}$ in evaluation of renal function, suggesting that it is a sensitive serum marker for the early assessment of kidney disease with diabetes $[11,12]$.

Retinol binding protein (RBP)-4, as a $21 \mathrm{kDa}$ protein, was initially known as a specific carrier for the delivery of retinol in circulation [13]. In the liver and adipose tissue, there was overexpression of RBP-4. Insulin resistance is a conspicuous characteristic of prediabetes mellitus (PDM) states (impaired fasting glucose (IFG) and/or impaired glucose tolerance (IGT)), and plays a crucial role in the development of T2DM. Increased serum RBP-4 levels have been reported in connection with IGT [14]. The relationship between RBP-4 and diabetes has been revealed in some studies; serum RBP-4 concentrations were negatively correlated with insulin resistance and development of T2DM [15-17]. However, the expression of RBP-4 in newly diagnosed T2DM and PDM and its association with insulin secretion and pancreas beta function are still unknown.

Homocysteine (HCY) is a sulfur-containing amino acid derived from methionine [18]. Previous studies have reported that increased HCY levels are a risk factor in relation to multiple diseases, including CV disease, neurodegenerative disease, and renal and vascular complications of diabetes [19-21]. Furthermore, there was an association of HCY and non-cardiac vascular diseases [22]. In vivo, hyperhomocysteinemia mice manifested glucose intolerance, insulin resistance, and impaired insulin signaling pathway [23]. As a risk factor, $\mathrm{HCY}$ is strongly linked to CV complications in T2DM. Nevertheless, there are still some controversial reports regarding the relationship of circulating HCY levels with insulin resistance in T2DM.

High-sensitivity C-reactive protein (hs-CRP), as one of the important biomarkers of inflammation, is mainly produced and released by the liver when stimulated by inflammatory factors
[24]. Serum concentration of hs-CRP was closely associated with systemic inflammatory conditions in the body. hs-CRP is closely linked to metabolic syndrome features; there was an increased hsCRP concentration in the IGT group compared to normal controls $[25,26]$. Studies have reported that hs-CRP concentrations were associated with insulin resistance and the development of diabetic nephropathy and coronary heart disease (CHD) risk [27-29].

In the present study, we aimed to investigate the expression levels of serum RBP-4, Cys C, HCY and hs-CRP in patients with newly diagnosed T2DM (NT2DM) by comparison with PDM subjects and healthy controls, and analyze their correlations with clinical manifestations and laboratory indexes.

\section{Material and methods}

\section{Study subjects and methods}

From June 2016 to December 2017, a total of 242 subjects (141 NT2DM patients, 48 PDM subjects and 53 normal controls) were recruited for the measurement of serum RBP-4, Cys C, HCY and hs-CRP concentrations. NT2DM patients and PDM subjects came from the Department of Endocrinology at the Second Hospital of Hefei City, when they first visited the DM clinic. The diagnosis of T2DM was established according to the American Diabetes Association diagnostic criteria 2018 [30]. PDM was defined as those without DM but a fasting plasma glucose (FPG) value $\geq 5.6 \mathrm{mmol} / \mathrm{l}$ and FPG $<6.9 \mathrm{mmol} / \mathrm{l}$ or a 2 -h plasma glucose $(2-\mathrm{h}$ $P G)$ value $\geq 7.8 \mathrm{mmol} / \mathrm{l}$ and $2-\mathrm{h} P G<11.1 \mathrm{mmol} / \mathrm{l}$ during a 75-g oral glucose tolerance test (OGTT) or glycated hemoglobin $\left(\mathrm{HbA}_{1 \mathrm{c}}\right) \geq 5.7 \%$ and $\mathrm{HbA}_{1 \mathrm{c}}$ $<6.4 \%$. Fifty-three healthy volunteers from a medical examination center, without liver or metabolic diseases, acute CV or cerebrovascular accidents, were included as normal controls. None of the study subjects were taking any medications during the study. Demographics, clinical manifestations and routine laboratory results were obtained from hospital medical records and then reviewed by experienced physicians.

\section{Standard protocol approvals and patient consent}

This study was approved by the Ethical Committee of the Second Hospital of Hefei City (Hefei, Anhui, China). All the study subjects provided informed consent to participate in this study.

All studies on humans described in the present manuscript were carried out with the approval of the responsible ethics committee and in accordance with national law and the Helsinki Declaration of 1975 (in its current, revised form). 


\section{Laboratory analyses}

After a fasting period of at least $10-12 \mathrm{~h}$ overnight, venous blood samples were collected. Fasting blood glucose (FBG), total cholesterol (TC), triglycerides (TGs), high-density lipoprotein cholesterol (HDL-C), low-density lipoprotein cholesterol ( $L D L-C)$, very low-density lipoprotein cholesterol (VLDL-C), alanine aminotransferase (ALT), alkaline phosphatase (ALP), aspartate aminotransferase (AST), $\gamma$-glutamyltransferase (GGT), lactate dehydrogenase (LDH), total bilirubin (TBIL), indirect bilirubin (IBIL), direct bilirubin (DBIL), $\mathrm{Cr}$ and uric acid (UA) were measured using a model 7600 automated bio-analyzer (Hitachi, Tokyo, Japan).

Blood pressure was measured after 15 min rest at the left arm in a sitting position using a sphygmomanometer. Body mass index (BMI) was calculated by dividing subjects' weight by their squared height $\left(\mathrm{kg} / \mathrm{m}^{2}\right)$.

Apolipoprotein (Apo)-A1 and Apo-B were measured by immunoturbidimetry (Roche/Cobas Integra Tinaquant, Roche Diagnostics).

$\mathrm{HCY}$ concentration was determined by the chemical luminescence method (Abbott Laboratories, Abbott Park, Illinois, USA).

The OGTT, with a standard oral glucose load (75 g), was performed after a 10-12-hour fast with venous blood sampling in the fasting state, then fasting blood glucose (FBG), 30 min blood glucose (BG), 60 min BG, 120 min (2 h) BG, fasting insulin, $30 \mathrm{~min}$ insulin, $60 \mathrm{~min}$ insulin, $120 \mathrm{~min}$ insulin, fasting C-peptide, $30 \mathrm{~min}$ insulin C-peptide, 60 min C-peptide and 120 min insulin C-peptide were measured. $\mathrm{HbA}_{1 \mathrm{c}}$ was measured using cation-exchange column chromatography on an automatic analyzer (Bio-Rad Company, Hercules, California, USA).

Surrogate markers of insulin sensitivity and secretion were computed, including homeostasis model of assessment of insulin resistance $(\mathrm{HOMA}-\mathrm{IR})=($ fasting serum insulin $(\mathrm{mmol} / \mathrm{ml}) \times$ FPG (mmol/l)/22.5) [31], HOMA $\beta$ cell function in$\operatorname{dex}($ HOMA $-\beta)=($ FINS $(\mu \mathrm{U} / \mathrm{ml}) \times 20 /$ FPG $(\mathrm{mmol} / \mathrm{l})$ - 3.5) [32] and the Matsuda index of insulin sensitivity (Matsuda ISI $=10000 /\left(\right.$ GluO*Ins0) ${ }^{1 / 2 *}$ (Glumean * Insmean) $^{1 / 2}$ ) [33].

\section{Enzyme-linked immunosorbent assay (ELISA) for serum RBP-4, Cys C and hs-CRP}

Blood samples were obtained from $5 \mathrm{ml}$ of whole blood of all study subjects and then frozen at $-80^{\circ} \mathrm{C}$ immediately after collection until assayed. Serum RBP-4, Cys C, and hs-CRP concentrations were determined by specific ELISA kits according to the manufacturer's recommendation (R\&D Systems, Inc).

\section{Statistical analysis}

Collected data were presented as mean \pm standard deviation (SD), or median (interquartile range, IQR) if they were not in normal distribution. One-way ANOVA or a nonparametric test (Kruskal-Wallis test) was applied to compare the statistical differences of continuous variables among study groups. The $\chi^{2}$ test or Fisher's exact test was used to analyze categorical variables. Correlation analyses were computed using Spearman's rank correlation coefficient. Receiver operating characteristic (ROC) analysis was performed and the area under the curve (AUC) was applied to assess specificity and sensitivity of using serum Cys $C$ and hs-CRP as biomarkers for T2DM or PDM. Statistical analysis was performed with the Statistical Package of Social Sciences (SPSS) 23.0 (SPSS Inc., Chicago, IL, USA) and MedCalc, version 11.4.2.0 (Mariakerke, Belgium). All results with a two-tailed $p<0.05$ were considered to be statistically significant.

\section{Results}

\section{Characteristics of the study population}

Table I shows the demographic and clinical characteristics of the study subjects. The distributions of age and gender in NT2DM, PDM and normal controls were not significantly different (all $p>0.05$ ). However, there were significant differences in BMI, waist circumference (WC), hip circumference, waist-to-hip ratio (WHR), systolic blood pressure (SBP), diastolic blood pressure (DBP), UA, IBIL, ALT, GGT, LDH, TC, TG, LDL-C, HDL-C and VLDL-C levels among NT2DM, PDM and NC groups. The pancreatic function was assessed in accordance to different time points of blood glucose, insulin, C-peptide, and the computed homeostasis model assessment of insulin resistance (HOMA-IR), homeostasis model assessment of $\beta$ cell function (HOMA- $\beta$ ) and Matsuda ISI (Table II).

\section{Comparison of RBP-4, Cys C, HCY and hs-CRP concentrations between NT2DM patients, PDM subjects and normal controls}

There were significant differences in serum Cys $C$ and hs-CRP levels among the three groups (both $p<0.05$ ). Compared to normal controls, there were significantly higher Cys $C$ and hs-CRP levels in the PDM group (both $p<0.001$ ), a significantly increased Cys $C$ level in the NT2DM group ( $p<0.001)$, but no significant differences in Cys $C$ and hs-CRP levels between NT2DM and PDM groups, and no significant differences in hs-CRP levels between NT2DM and normal controls. In addition, the results indicated that there were no sig- 
Table I. Clinical characteristics of study subjects

\begin{tabular}{|c|c|c|c|c|}
\hline Parameters & NT2DM $(n=141)$ & $\operatorname{PDM}(n=48)$ & NC $(n=53)$ & $P$-value \\
\hline Age [years] & $50.8 \pm 10.1$ & $49.7 \pm 13.0$ & $47.0 \pm 8.0$ & 0.078 \\
\hline Gender (female/male) & $69 / 72$ & $23 / 25$ & $25 / 28$ & 0.974 \\
\hline $\mathrm{BMI}\left[\mathrm{kg} / \mathrm{cm}^{2}\right]$ & $26.5(25.4,27.7)$ & $25.7(24.1,26.8)$ & $23.6(22.1,24.6)$ & $<0.001$ \\
\hline$W C[\mathrm{~cm}]$ & $88.72 \pm 9.94$ & $91.87 \pm 9.00$ & $83.08 \pm 7.81$ & $<0.001$ \\
\hline $\mathrm{HIP}[\mathrm{cm}]$ & $92.06 \pm 8.14$ & $98.63 \pm 8.39$ & $95.19 \pm 6.96$ & $<0.001$ \\
\hline WHR & $0.95(0.92,1.04)$ & $0.90(0.88,0.94)$ & $0.86(0.83,0.88)$ & $<0.001$ \\
\hline $\mathrm{SBP}[\mathrm{mm} \mathrm{Hg}]$ & $135 \pm 16$ & $136 \pm 16$ & $125 \pm 14$ & 0.001 \\
\hline $\mathrm{DBP}[\mathrm{mm} \mathrm{Hg}]$ & $83 \pm 11$ & $83 \pm 11$ & $77 \pm 10$ & 0.001 \\
\hline Urea nitrogen [mmol/l] & $5.10 \pm 1.48$ & $5.09 \pm 1.56$ & $4.82 \pm 1.18$ & 0.464 \\
\hline $\mathrm{Cr}[\mu \mathrm{mol} / \mathrm{l}]$ & $57.97 \pm 14.37$ & $59.98 \pm 14.20$ & $62.85 \pm 13.80$ & 0.101 \\
\hline $\mathrm{UA}[\mu \mathrm{mol} / \mathrm{l}]$ & $342.04 \pm 63.36$ & $319.25 \pm 75.46$ & $294.15 \pm 54.47$ & $<0.001$ \\
\hline TBIL $[\mu \mathrm{mol} / / \mathrm{l}]$ & $15.9(13.2,21.2)$ & $15.7(11.5,20.5)$ & $13.6(11.3,16.6)$ & 0.074 \\
\hline $\mathrm{DBIL}[\mu \mathrm{mol} / \mathrm{l}]$ & $4.2(3.3,5.5)$ & $3.7(2.7,5.1)$ & $4.2(3.0,5.1)$ & 0.540 \\
\hline $\mid \mathrm{BIL}[\mu \mathrm{mol} / \mathrm{I}]$ & $11.9(9.6,16.3)$ & $12.0(8.4,15.1)$ & $9.6(7.9,12.5)$ & 0.045 \\
\hline ALP [U/I $]$ & $76 \pm 21$ & $76 \pm 21$ & $71 \pm 22$ & 0.289 \\
\hline AST [U/I] & $20(16,28)$ & $24(18,29)$ & $19(16,24)$ & 0.019 \\
\hline $\operatorname{ALT}[\mathrm{U} / \mathrm{I}]$ & $35(32,38)$ & $31(26,36)$ & $18(13,27)$ & $<0.001$ \\
\hline GGT [U/I] & $36(26,54)$ & $34(19,68)$ & $23(16,34)$ & 0.007 \\
\hline $\mathrm{LDH}[\mathrm{U} / \mathrm{I}]$ & $166 \pm 33$ & $189 \pm 38$ & $184 \pm 32$ & $<0.001$ \\
\hline $\mathrm{TG}[\mathrm{mmol} / \mathrm{l}]$ & $2.4 \pm 0.6$ & $1.9 \pm 0.7$ & $1.4 \pm 0.6$ & $<0.001$ \\
\hline $\mathrm{TC}[\mathrm{mmol} / \mathrm{l}]$ & $5.4 \pm 0.3$ & $4.8 \pm 0.2$ & $4.1 \pm 0.4$ & $<0.001$ \\
\hline $\mathrm{HDL}-\mathrm{C}[\mathrm{mmol} / \mathrm{l}]$ & $1.4 \pm 0.3$ & $1.5 \pm 0.3$ & $1.6 \pm 0.4$ & $<0.001$ \\
\hline LDL-C [mmol/l] & $3.3 \pm 0.4$ & $2.9 \pm 0.7$ & $2.2 \pm 0.2$ & $<0.001$ \\
\hline VLDL-C [mmol/ll] & $0.4(0.3,0.6)$ & $0.3(0.2,0.5)$ & $0.2(0.1,0.4)$ & 0.003 \\
\hline Apo-B [g/l] & $0.9(0.7,1.1)$ & $0.9(0.7,1.0)$ & $0.9(0.8,1.0)$ & 0.403 \\
\hline Apo-A1 [g/l] & $1.1 \pm 0.2$ & $1.2 \pm 0.2$ & $1.2 \pm 0.2$ & $<0.001$ \\
\hline
\end{tabular}

Apo-A1 - apolipoprotein-A1, Apo-B - apolipoprotein-B, ALP - alkaline phosphatase, AST - aspartate aminotransferase, ALT - alanine transaminase, $B M I$ - body mass index, $C r$-creatine, DBIL - direct bilirubin, DBP - diastolic blood pressure, FBG - fasting blood glucose, GGT - $\gamma$-glutamyltransferase, HDL-C - high-density lipoprotein cholesterol, IBIL - indirect bilirubin, LDH - lactate dehydrogenase, LDL-C low-density lipoprotein cholesterol, NT2DM - newly diagnosed type 2 diabetes mellitus, NC - normal control, PDM - prediabetes mellitus, SBP - systolic blood pressure, TC - total cholesterol, TG - triglycerides, TBIL - total bilirubin, UA - uric acid, VLDL-C - very low-density lipoprotein cholesterol, WC - waist circumference, WHR - waist-to-hip ratio.

nificant differences in serum RBP-4 and HCY levels among NT2DM, PDM and NC groups (all $p>0.05$ ) (Table III).

\section{Correlations of Cys C levels with clinical and laboratory parameters among study groups}

The results of univariate correlation analysis showed that Cys $C$ concentrations were significantly correlated with age, WHR, FBG, C-peptide (0 min, $120 \mathrm{~min}$ ), urea nitrogen, Cr, UA, TBIL, IBIL,
AST, HDL-C, VLDL-C and HOMA- $\beta$ in the NT2DM group (all $p<0.05$ ), and correlated with SBP, $\mathrm{Cr}, \mathrm{UA}$ and LDL-C in the PDM group (all $p<0.05$ ). Moreover, there was a significant association of serum Cys $C$ levels with age, BMI, SBP, FBG, C-peptide (0 min, $120 \mathrm{~min}$ ), Cr, UA, DBIL, ALT, AST, GGT, TG, TC, HDL-C, VLDL-C, HOMA-IR and Matsuda ISI in normal controls (all $p<0.05$ ). However, no significant correlations of Cys $C$ with other clinical and quantitative laboratory parameters were observed (all $p>0.05$ ) (Table IV). 
Table II. Evaluation of pancreatic function of study population

\begin{tabular}{|c|c|c|c|c|}
\hline Parameter & $\begin{array}{l}\text { NT2DM } \\
(n=141)\end{array}$ & $\begin{array}{c}\text { PDM } \\
(n=48)\end{array}$ & $\begin{array}{l}\text { Normal control } \\
\quad(n=53)\end{array}$ & $P$-value \\
\hline FBG [mmol/l] & $7.44 \pm 1.07$ & $5.54 \pm 0.65$ & $4.84 \pm 0.62$ & $<0.001$ \\
\hline $30 \mathrm{~min}$ BG (OGTT) $[\mathrm{mmol} / \mathrm{l}]$ & $13.19 \pm 1.71$ & $10.57 \pm 1.80$ & $8.78 \pm 1.63$ & $<0.001$ \\
\hline $60 \mathrm{~min}$ BG (OGTT) [mmol/l] & $15.77 \pm 1.85$ & $11.69 \pm 2.15$ & $8.06 \pm 2.49$ & $<0.001$ \\
\hline $120 \min B G(O G T T)[\mathrm{mmol} / \mathrm{l}]$ & $16.90(14.00,17.88)$ & $8.93(8.05,9.75)$ & $6.05(5.34,6.71)$ & $<0.001$ \\
\hline Insulin (OGTT 0 min) [mU/l] & $6.31(5.24,8.47)$ & $7.77(6.05,8.70)$ & $6.63(5.54,8.26)$ & 0.021 \\
\hline Insulin (OGTT $30 \mathrm{~min}$ ) [mU/l] & $16.81(10.41,28.04)$ & $43.99(30.14,63.56)$ & $51.80(33.60,71.89)$ & $<0.001$ \\
\hline Insulin (OGTT $60 \mathrm{~min}$ ) [mU/l] & $23.88(16.22,38.08)$ & $65.67(47.54,80.36)$ & $55.08(38.46,75.87)$ & $<0.001$ \\
\hline Insulin (OGTT $120 \mathrm{~min}$ ) [mU/l] & $28.86(17.24,48.99)$ & $64.72(42.09,89.68)$ & $34.17(21.53,47.12)$ & $<0.001$ \\
\hline 0 min C-peptide (OGTT) [ng/ml] & $1.48 \pm 0.70$ & $1.76 \pm 0.66$ & $1.26 \pm 0.56$ & 0.002 \\
\hline 30 min C-peptide (OGTT) [ng/ml] & $2.46(1.73,3.77)$ & $5.14(3.77,6.36)$ & $4.70(3.15,5.99)$ & $<0.001$ \\
\hline 60 min C-peptide (OGTT) [ng/ml] & $3.61(2.29,5.31)$ & $7.78(5.88,8.77)$ & $6.20(4.56,8.59)$ & $<0.001$ \\
\hline 120 min C-peptide (OGTT) [ng/ml] & $5.50 \pm 3.26$ & $8.69 \pm 2.95$ & $5.59 \pm 2.33$ & $<0.001$ \\
\hline $\mathrm{HbA}_{1 c}(\%)$ & $7.91 \pm 0.42$ & $6.06 \pm 0.28$ & $5.12 \pm 0.31$ & $<0.001$ \\
\hline HOMA-IR & $2.20(1.73,2.78)$ & $1.82(1.45,2.22)$ & $1.44(1.19,1.86)$ & $<0.001$ \\
\hline HOMA- $\beta$ & $32.89(24.32,45.60)$ & $81.09(59.16,109.50)$ & $117.11(74.55,158.77)$ & $<0.001$ \\
\hline Matsuda ISI & $93.38 \pm 38.31$ & $78.76 \pm 24.14$ & $114.64 \pm 44.28$ & $<0.001$ \\
\hline
\end{tabular}

$B G$ - blood glucose, FBG - fasting blood glucose, HOMA-IR - homeostasis model assessment of insulin resistance, HOMA- $\beta$ - HOMA beta cell function index, $H b A_{1 c}$ - glycated hemoglobin, OGTT - oral glucose tolerance test, PDM - prediabetes mellitus, NT2DM - newly diagnosed type 2 diabetes mellitus.

Table III. Comparison of RBP-4, Cys C, HCY and hs-CRP levels among three groups

\begin{tabular}{|lcccc|}
\hline Parameter & NT2DM $(n=141)$ & PDM $(n=48)$ & NC $(n=53)$ & $P$-value \\
\hline RBP-4 $[\mu \mathrm{g} / \mathrm{ml}]$ & $49.00(39.95,56.95)$ & $47.55(40.65,63.63)$ & $50.90(43.45,60.05)$ & 0.461 \\
\hline Cys C $[\mathrm{mg} / \mathrm{ml}]$ & $0.87(0.75,1.00)^{*}$ & $0.83(0.77,1.05)^{\star *}$ & $0.76(0.65,0.86)$ & $<0.001$ \\
\hline HCY $[\mu \mathrm{mol} / \mathrm{l}]$ & $11.30(9.40,14.35)$ & $11.60(10.50,14.03)$ & $10.90(8.65,12.95)$ & 0.350 \\
\hline hs-CRP $[\mu \mathrm{g} / \mathrm{ml}]$ & $0.55(0.40,0.74)$ & $0.61(0.50,0.99)^{* *}$ & $0.50(0.40,0.57)$ & 0.006 \\
\hline
\end{tabular}

"Significant difference in NT2DM versus NC. " Significant difference in PDM versus NC. RBP-4 - retinol binding protein-4, Cys C-cystatin C, $\mathrm{HCY}$ - homocysteine, $\mathrm{Hs}$-CRP - high-sensitivity C-reactive protein, PDM - prediabetes mellitus, NT2DM-newly diagnosed type 2 diabetes mellitus, NC - normal control.

Correlations of hs-CRP levels with clinical and laboratory parameters among study groups

Univariate correlation analysis demonstrated that serum hs-CRP levels positively associated with BMI, ALP and HOMA- $\beta$ in the NT2DM group (all $p<0.05$ ), and positively associated with $2 \mathrm{~h}$ BG, but negatively associated with GGT in the PDM group (all $p<0.05$ ). In addition, in normal controls, there was a significant positive association of serum hs-CRP levels with C-peptide (0 min, $120 \mathrm{~min}$ ), UA, GGT, and ALP and a negative association with HDL-C and Apo-A1 (all $p<0.05$ ). However, no significant correlations of hs-CRP with other clinical and quantitative laboratory parameters were found (all $p>0.05$ ) (Table V).

\section{Correlations of HCY levels with clinical and laboratory parameters among study groups}

In the NT2DM group, HCY level correlated with $\mathrm{Cr}$, UA, TBIL, DBIL, IBIL, glutamic-pyruvic transaminase (GPT), AST, LDH and Apo-A1 (all $p<0.05$ ), while in the PDM group, HCY level positively associated with WHR, but negatively associated with ALP (all $p<0.05$ ). Furthermore, we found that HCY level positively correlated with SBP, DBP, UA, ALP, LDH and HDL-C in normal controls (all $p<0.05$ ). No significant correlations of $\mathrm{HCY}$ with other clinical and quantitative laboratory parameters were observed (all $p>0.05$ ) (Table VI). 
Table IV. Association of Cys C level with demographic and clinical characteristics

\begin{tabular}{|c|c|c|c|c|c|c|}
\hline \multirow[t]{2}{*}{ Parameter } & \multicolumn{2}{|c|}{ NT2DM $(n=141)$} & \multicolumn{2}{|c|}{$\operatorname{PDM}(n=48)$} & \multicolumn{2}{|c|}{ Normal control $(n=53)$} \\
\hline & $r$ & $p$ & $r$ & $p$ & $r$ & $p$ \\
\hline Age & 0.196 & 0.020 & 0.056 & 0.708 & 0.297 & 0.031 \\
\hline BMI & 0.117 & 0.166 & 0.177 & 0.228 & 0.361 & 0.008 \\
\hline WHR & 0.261 & 0.002 & 0.254 & 0.082 & 0.260 & 0.060 \\
\hline SBP & 0.115 & 0.175 & 0.346 & 0.016 & 0.542 & $<0.001$ \\
\hline DBP & 0.087 & 0.303 & 0.196 & 0.181 & 0.268 & 0.052 \\
\hline FBG & -0.246 & 0.003 & 0.080 & 0.591 & 0.303 & 0.027 \\
\hline 120 min BG (OGTT) & -0.141 & 0.095 & -0.029 & 0.843 & 0.166 & 0.234 \\
\hline Insulin (OGTT 0 min) & 0.159 & 0.060 & 0.186 & 0.207 & 0.261 & 0.060 \\
\hline Insulin (OGTT 120 min) & 0.129 & 0.129 & 0.134 & 0.363 & -0.004 & 0.979 \\
\hline C-peptide 0 min (OGTT) & 0.253 & 0.002 & 0.169 & 0.250 & 0.583 & $<0.001$ \\
\hline C-peptide $120 \min$ (OGTT) & 0.173 & 0.040 & 0.190 & 0.195 & 0.339 & 0.013 \\
\hline Urea nitrogen & 0.186 & 0.027 & 0.131 & 0.376 & 0.220 & 0.113 \\
\hline $\mathrm{Cr}$ & 0.462 & $<0.001$ & 0.467 & 0.001 & 0.424 & 0.002 \\
\hline UA & 0.192 & 0.023 & 0.409 & 0.004 & 0.468 & $<0.001$ \\
\hline TBIL & 0.210 & 0.013 & 0.181 & 0.219 & -0.160 & 0.253 \\
\hline DBIL & 0.157 & 0.063 & 0.168 & 0.253 & -0.330 & 0.016 \\
\hline IBIL & 0.201 & 0.017 & 0.185 & 0.208 & -0.007 & 0.963 \\
\hline ALT & 0.144 & 0.088 & 0.160 & 0.277 & 0.506 & $<0.001$ \\
\hline AST & 0.246 & 0.003 & 0.245 & 0.093 & 0.438 & 0.001 \\
\hline GGT & 0.108 & 0.203 & 0.271 & 0.063 & 0.359 & 0.008 \\
\hline ALP & 0.164 & 0.052 & 0.020 & 0.895 & 0.222 & 0.110 \\
\hline LDH & 0.072 & 0.397 & 0.188 & 0.200 & 0.136 & 0.330 \\
\hline TG & 0.021 & 0.805 & 0.006 & 0.970 & 0.481 & $<0.001$ \\
\hline TC & -0.021 & 0.808 & 0.022 & 0.883 & 0.365 & 0.007 \\
\hline HDL-C & -0.255 & 0.002 & -0.126 & 0.393 & -0.334 & 0.015 \\
\hline LDL-C & 0.024 & 0.781 & 0.293 & 0.043 & 0.103 & 0.461 \\
\hline VLDL-C & 0.172 & 0.042 & 0.096 & 0.516 & 0.478 & $<0.001$ \\
\hline Apo-B & -0.091 & 0.284 & 0.268 & 0.065 & 0.113 & 0.420 \\
\hline Apo-A1 & -0.089 & 0.294 & -0.192 & 0.192 & -0.244 & 0.079 \\
\hline HOMA-IR & 0.082 & 0.332 & 0.205 & 0.163 & 0.354 & 0.009 \\
\hline HOMA- $\beta$ & 0.250 & 0.003 & 0.039 & 0.791 & -0.120 & 0.392 \\
\hline ISI Matsudo & -0.123 & 0.146 & -0.153 & 0.300 & -0.301 & 0.028 \\
\hline
\end{tabular}

Apo-A1 - apolipoprotein-A1, Apo-B - apolipoprotein-B, ALP - alkaline phosphatase, ALT - alanine transaminase, AST - aspartate aminotransferase, $B M I$ - body mass index, $B U$ - blood urea, $C r$ - creatine, Cys $C$ - cystatin $C$, DBIL - direct bilirubin, DBP - diastolic blood pressure, FBG - fasting blood glucose, GGT - $\gamma$-glutamyltransferase, HDL-C - high-density lipoprotein cholesterol, IBIL - indirect bilirubin, $L D H$ - lactate dehydrogenase, LDL-C - low-density lipoprotein cholesterol, NT2DM - newly diagnosed type 2 diabetes mellitus, NC - normal control, PDM - prediabetes mellitus, SBP - systolic blood pressure, TC - total cholesterol, TG - triglycerides, TBIL - total bilirubin, UA - uric acid, VLDL-C - very low-density lipoprotein cholesterol, WC - waist circumference, WHR - waist-to-hip ratio. 
Association of retinol binding protein-4, cystatin C, homocysteine and high-sensitivity C-reactive protein levels in patients with newly diagnosed type 2 diabetes mellitus

Table V. Association of hs-CRP level with demographic and clinical characteristics

\begin{tabular}{|c|c|c|c|c|c|c|}
\hline \multirow[t]{2}{*}{ Parameters } & \multicolumn{2}{|c|}{ NT2DM $(n=141)$} & \multicolumn{2}{|c|}{$\operatorname{PDM}(n=48)$} & \multicolumn{2}{|c|}{ Normal control $(n=53)$} \\
\hline & $r$ & $p$ & $r$ & $p$ & $r$ & $p$ \\
\hline Age & -0.073 & 0.393 & 0.046 & 0.756 & -0.094 & 0.504 \\
\hline BMI & 0.258 & 0.002 & 0.267 & 0.067 & 0.130 & 0.352 \\
\hline WHR & 0.117 & 0.169 & 0.098 & 0.506 & 0.235 & 0.091 \\
\hline SBP & 0.009 & 0.913 & 0.100 & 0.501 & 0.160 & 0.253 \\
\hline DBP & 0.113 & 0.180 & 0.156 & 0.290 & -0.031 & 0.824 \\
\hline FBG & -0.088 & 0.297 & 0.031 & 0.837 & 0.041 & 0.769 \\
\hline 120 min BG (OGTT) & 0.055 & 0.520 & -0.290 & 0.046 & -0.076 & 0.591 \\
\hline Insulin (OGTT 0 min) & 0.139 & 0.101 & 0.103 & 0.485 & 0.242 & 0.081 \\
\hline Insulin (OGTT 120 min) & 0.034 & 0.691 & -0.029 & 0.844 & 0.088 & 0.531 \\
\hline C-peptide 0 min (OGTT) & 0.124 & 0.142 & 0.279 & 0.055 & 0.430 & 0.001 \\
\hline C-peptide 120 min (OGTT) & 0.057 & 0.503 & 0.224 & 0.127 & 0.291 & 0.034 \\
\hline Urea nitrogen & -0.024 & 0.780 & -0.028 & 0.848 & -0.080 & 0.567 \\
\hline $\mathrm{Cr}$ & -0.025 & 0.765 & 0.047 & 0.749 & 0.110 & 0.433 \\
\hline UA & 0.130 & 0.125 & 0.251 & 0.085 & 0.273 & 0.048 \\
\hline TBIL & 0.017 & 0.841 & -0.026 & 0.859 & -0.002 & 0.989 \\
\hline DBIL & -0.079 & 0.355 & 0.000 & 0.997 & -0.190 & 0.172 \\
\hline IBIL & 0.047 & 0.581 & -0.048 & 0.747 & 0.043 & 0.762 \\
\hline ALT & 0.136 & 0.109 & 0.057 & 0.699 & -0.005 & 0.970 \\
\hline AST & 0.019 & 0.823 & 0.174 & 0.238 & -0.034 & 0.808 \\
\hline GGT & 0.113 & 0.181 & 0.312 & 0.031 & 0.302 & 0.028 \\
\hline ALP & 0.179 & 0.033 & 0.074 & 0.616 & 0.412 & 0.002 \\
\hline LDH & 0.086 & 0.312 & 0.105 & 0.477 & 0.151 & 0.281 \\
\hline TG & -0.039 & 0.649 & -0.023 & 0.879 & 0.243 & 0.080 \\
\hline TC & -0.035 & 0.680 & 0.247 & 0.091 & 0.080 & 0.568 \\
\hline HDL-C & -0.100 & 0.237 & -0.194 & 0.187 & -0.351 & 0.010 \\
\hline LDL-C & -0.040 & 0.640 & 0.261 & 0.073 & -0.084 & 0.549 \\
\hline VLDL-C & 0.086 & 0.309 & 0.084 & 0.572 & 0.206 & 0.139 \\
\hline Apo-B & -0.010 & 0.910 & -0.026 & 0.862 & -0.174 & 0.212 \\
\hline Apo-A1 & -0.161 & 0.056 & -0.243 & 0.096 & -0.340 & 0.013 \\
\hline HOMA-IR & 0.114 & 0.176 & 0.119 & 0.422 & 0.216 & 0.121 \\
\hline HOMA- $\beta$ & 0.181 & 0.032 & 0.054 & 0.717 & 0.053 & 0.706 \\
\hline$|S|$ & -0.104 & 0.220 & 0.022 & 0.883 & -0.268 & 0.053 \\
\hline
\end{tabular}

Apo-A1 - apolipoprotein-A1, Apo-B - apolipoprotein-B, ALP - alkaline phosphatase, ALT - alanine transaminase, AST - aspartate aminotransferase, $B M I$ - body mass index, $B U$ - blood urea, $\mathrm{Cr}$ - creatine, DBIL - direct bilirubin, DBP - diastolic blood pressure, $F B G$ - fasting blood glucose, GGT - $\gamma$-glutamyltransferase, hs-CRP - high-sensitivity C-reactive protein, HDL-C - high-density lipoprotein cholesterol, IBIL - indirect bilirubin, LDH - lactate dehydrogenase, LDL-C - low-density lipoprotein cholesterol, NT2DM - newly diagnosed type 2 diabetes mellitus, NC - normal control, PDM - prediabetes mellitus, SBP - systolic blood pressure, TC - total cholesterol, $T G$ - triglycerides, TBIL - total bilirubin, UA - uric acid, VLDL-C - very low-density lipoprotein cholesterol, WC - waist circumference, WHR - waist-to-hip ratio. 
Table VI. Association of HCY level with demographic and clinical characteristics

\begin{tabular}{|c|c|c|c|c|c|c|}
\hline \multirow[t]{2}{*}{ Parameter } & \multicolumn{2}{|c|}{ NT2DM $(n=141)$} & \multicolumn{2}{|c|}{$\operatorname{PDM}(n=48)$} & \multicolumn{2}{|c|}{ Normal control $(n=53)$} \\
\hline & $r$ & $p$ & $r$ & $p$ & $r$ & $p$ \\
\hline Age & 0.086 & 0.312 & -0.039 & 0.793 & 0.137 & 0.329 \\
\hline BMI & 0.060 & 0.478 & 0.018 & 0.901 & 0.148 & 0.289 \\
\hline WHR & 0.160 & 0.057 & 0.405 & 0.004 & 0.009 & 0.950 \\
\hline SBP & -0.015 & 0.857 & 0.089 & 0.545 & 0.395 & 0.003 \\
\hline DBP & 0.045 & 0.597 & 0.107 & 0.471 & 0.377 & 0.005 \\
\hline FBG & -0.103 & 0.223 & -0.091 & 0.539 & 0.015 & 0.915 \\
\hline 120 min BG (OGTT) & 0.003 & 0.968 & -0.073 & 0.621 & -0.024 & 0.866 \\
\hline Insulin (OGTT 0 min) & 0.075 & 0.375 & 0.062 & 0.674 & -0.051 & 0.716 \\
\hline Insulin (OGTT 120 min) & 0.068 & 0.420 & 0.076 & 0.608 & -0.127 & 0.364 \\
\hline C-peptide 0 min (OGTT) & -0.014 & 0.872 & -0.027 & 0.854 & 0.163 & 0.243 \\
\hline C-peptide $120 \min$ (OGTT) & 0.001 & 0.989 & 0.168 & 0.255 & 0.104 & 0.457 \\
\hline Urea nitrogen & 0.069 & 0.413 & -0.032 & 0.828 & -0.026 & 0.852 \\
\hline $\mathrm{Cr}$ & 0.302 & $<0.001$ & 0.233 & 0.111 & 0.185 & 0.185 \\
\hline UA & 0.189 & 0.025 & 0.185 & 0.209 & 0.337 & 0.014 \\
\hline TBIL & 0.229 & 0.006 & 0.050 & 0.735 & -0.041 & 0.768 \\
\hline DBIL & 0.238 & 0.004 & 0.119 & 0.419 & -0.095 & 0.500 \\
\hline IBIL & 0.206 & 0.014 & 0.017 & 0.910 & -0.029 & 0.835 \\
\hline GPT & 0.207 & 0.014 & 0.180 & 0.222 & 0.210 & 0.131 \\
\hline AST & 0.219 & 0.009 & 0.149 & 0.312 & 0.265 & 0.056 \\
\hline ALT & 0.044 & 0.607 & 0.280 & 0.054 & 0.178 & 0.202 \\
\hline ALP & 0.160 & 0.058 & -0.394 & 0.006 & 0.365 & 0.007 \\
\hline LDH & 0.195 & 0.021 & 0.278 & 0.056 & 0.429 & 0.001 \\
\hline TG & 0.005 & 0.949 & -0.133 & 0.367 & 0.230 & 0.097 \\
\hline $\mathrm{TC}$ & 0.024 & 0.777 & 0.169 & 0.250 & 0.254 & 0.067 \\
\hline HDL-C & -0.265 & 0.001 & -0.097 & 0.512 & -0.273 & 0.048 \\
\hline LDL-C & -0.134 & 0.114 & 0.015 & 0.921 & 0.069 & 0.623 \\
\hline VLDL-C & 0.012 & 0.883 & -0.005 & 0.974 & 0.183 & 0.190 \\
\hline Apo-B & -0.093 & 0.273 & 0.188 & 0.200 & 0.060 & 0.669 \\
\hline Apo-A1 & -0.166 & 0.049 & -0.009 & 0.950 & -0.184 & 0.188 \\
\hline HOMA-IR & 0.030 & 0.724 & 0.047 & 0.749 & -0.057 & 0.687 \\
\hline HOMA- $\beta$ & 0.108 & 0.204 & 0.121 & 0.413 & -0.040 & 0.774 \\
\hline ISI & -0.082 & 0.336 & -0.084 & 0.572 & 0.037 & 0.792 \\
\hline
\end{tabular}

Apo-A1 - apolipoprotein-A1, Apo-B - apolipoprotein-B, ALP - alkaline phosphatase, ALT - alanine transaminase, AST - aspartate aminotransferase, $B M I$ - body mass index, $B U$ - blood urea, $\mathrm{Cr}$ - creatine, DBIL - direct bilirubin, DBP - diastolic blood pressure, FBG - fasting blood glucose, GPT - glutamic-pyruvic transaminase, HCY - homocysteine, HDL-C - high-density lipoprotein cholesterol, $I B I L$ - indirect bilirubin, $L D H$ - lactate dehydrogenase, $L D L-C$ - low-density lipoprotein cholesterol, NT2DM - newly diagnosed type 2 diabetes mellitus, NC - normal control, PDM - prediabetes mellitus, SBP - systolic blood pressure, TC - total cholesterol, TG - triglycerides, TBIL - total bilirubin, UA - uric acid, VLDL-C - very low-density lipoprotein cholesterol, WC - waist circumference, WHR-waist-to-hip ratio. 
Correlations of RBP-4 levels with clinical and laboratory parameters among study groups

The correlation analysis demonstrated that serum RBP-4 concentration was correlated positively with $\mathrm{Cr}$, UA, ALT, VLDL-C and Apo-B in the NT2DM group (all $p<0.05$ ), positively associated with $\mathrm{Cr}$ UA, DBIL and ALT in the PDM group (all $p<0.05$ ), and there was a positive association with urea nitrogen, IBIL, GPT, AST and VLDL-C in the normal control group (all $p<0.05$ ). However, no significant correlations of RBP-4 with other clinical and quantitative laboratory parameters were observed (all $p>0.05$ ) (Table VII).

\section{Predictive accuracy of Cys $C$ and hs-CRP for diagnostic biomarkers of NT2DM}

An ROC curve was drawn to determine the diagnostic performance of Cys $C$ and hs-CRP as NT2DM biomarkers. The Cys $C$ had an area under the curve (AUC) value of $0.683(95 \% \mathrm{Cl}: 0.612-$ 0.747 ), with $58.16 \%$ sensitivity and $75.47 \%$ specificity (Figure $1 \mathrm{~A}$ ). For hs-CRP, the AUC value was 0.578 (95\% Cl: 0.505-0.649), with $46.81 \%$ sensitivity and $77.36 \%$ specificity (Figure $1 \mathrm{~B}$ ).

Predictive accuracy of Cys C and hs-CRP for diagnostic biomarkers of PDM

The diagnostic performance of Cys $C$ and hsCRP as PDM biomarkers was assessed by ROC curve and AUC. The AUC value of Cys C is 0.696 (95\% Cl: $0.597-0.784)$, with $75.00 \%$ sensitivity and $54.72 \%$ specificity (Figure $2 \mathrm{~A}$ ). Moreover, hsCRP had an AUC value of 0.694 (95\% Cl: 0.5950.782 ), with $81.13 \%$ sensitivity and $56.25 \%$ specificity (Figure $2 \mathrm{~B}$ ).

\section{Discussion}

Although RBP-4, Cys C, HCY and hs-CRP have been studied in many metabolic diseases, there are very few studies regarding the expression of RBP-4, Cys C, HCY and hs-CRP and their association with NT2DM. In the present study, we investigated the serum RBP-4, Cys C, HCY and hs-CRP concentrations in NT2DM and their relations with clinical and laboratory features. The current study demonstrated that, in comparison to healthy controls, there were significantly increased Cys $C$ levels in NT2DM and significantly elevated Cys $\mathrm{C}$ and hs-CRP levels in PDM. Both the $\mathrm{Cr}$ and UA were correlated with Cys C concentration among NT2DM, PDM and normal controls. Furthermore, hs-CRP level was correlated with BMI, ALP and HOMA- $\beta$ in the NT2DM group, and was associated with $2 \mathrm{~h}$ BG and GGT in the PDM group. In normal controls, serum hs-CRP level cor- related with C-peptide (0 min, 120 min), UA, GGT, ALP, HDL-C and Apo-A1. However, we did not find any differences of RBP-4 and HCY concentration among NT2DM, PDM and normal controls.

As we know, patients with T2DM have a high incidence of renal involvement $[34,35]$. Cys $C$ is a cysteine protease inhibitor that is produced at a constant rate by all nucleated cells and freely filtered by glomeruli owing to its low molecular weight; thus, it can freely pass through the glomeruli and be reabsorbed in proximal tubules, followed by complete catabolism without being affected by features influencing $\mathrm{Cr}$ level $[11,36]$. Previous studies have revealed that there was a higher Cys $C$ level in T2DM patients than in controls, and it was associated with renal dysfunction $[12,37]$. Consistent with prior studies, we found that in NT2DM patients, there was a higher Cys C level than in healthy controls, and serum Cys $C$ concentrations were positively associated with age, WHR, C-peptide (0 min, $120 \mathrm{~min}$ ), urea nitrogen, $\mathrm{Cr}$, UA, TBIL, IBIL, AST, HDL-C, VLDL-C and HOMA- $\beta$, and were negatively associated with FBG and HDL-C. In addition, there was a higher Cys $C$ level in PDM than in the control group. The increased Cys $C$ level was correlated with SBP, $\mathrm{Cr}$, UA and LDL-C. It has been reported that the Cys $C$ level is able to increase at the stage of PDM, and a study of 83 patients with T2DM showed that Cys $C$ is a good marker of incipient renal disease, which may be because minimal glomerular damage could result in a significant increase of serum Cys C level, causing disease progression [38]. Therefore, early detection of Cys $C$ not only helps to predict the possible potential diabetes mellitus risk, but also benefits in identification of early renal function disorder of T2DM.

Hs-CRP has been applied in clinical settings to monitor chronic and acute inflammatory conditions [39]. As a systemic marker, it is extremely sensitive to inflammatory condition and tissue damage and plays a crucial part in atherosclerosis and thus causes CV disease [40, 41]. Serum hs-CRP is positively associated with the metabolic syndrome and has been acknowledged to be an independent risk factor for development of diabetic neuropathy, diabetic foot ulcers and CV complications [42, 43]. Recently, Aryan et al. performed a larger sample population-based study to evaluate the predicted value of hs-CRP for complications of T2DM. The results showed that serum hs-CRP associated with the risk of coronary heart disease $(\mathrm{CHD})$ (hazard ratio $(\mathrm{HR})=1.028,95 \% \mathrm{Cl}$ : 1.024-1.032), diabetic neuropathy $(H R=1.025$, $95 \% \mathrm{Cl}: 1.021-1.029)$, diabetic retinopathy ( $\mathrm{HR}=$ $1.037,95 \% \mathrm{Cl}: 1.030-1.043$ and diabetic kidney disease $(H R=1.035,95 \% \mathrm{Cl}: 1.027-1.043)$ [44]. In the present study, our results showed a signifi- 
Table VII. Association of RBP-4 level with demographic and clinical characteristics

\begin{tabular}{|c|c|c|c|c|c|c|}
\hline \multirow[t]{2}{*}{ Parameter } & \multicolumn{2}{|c|}{ NT2DM $(n=141)$} & \multicolumn{2}{|c|}{$\operatorname{PDM}(n=48)$} & \multicolumn{2}{|c|}{ Normal control $(n=53)$} \\
\hline & $r$ & $p$ & $r$ & $p$ & $r$ & $p$ \\
\hline Age & 0.013 & 0.876 & 0.058 & 0.695 & 0.028 & 0.844 \\
\hline BMI & 0.110 & 0.195 & 0.098 & 0.509 & -0.182 & 0.192 \\
\hline WHR & -0.013 & 0.874 & 0.051 & 0.733 & -0.076 & 0.590 \\
\hline SBP & -0.089 & 0.295 & 0.085 & 0.565 & 0.015 & 0.917 \\
\hline DBP & -0.026 & 0.760 & 0.254 & 0.082 & -0.028 & 0.844 \\
\hline FBG & 0.065 & 0.443 & 0.052 & 0.726 & -0.077 & 0.582 \\
\hline 120 min BG (OGTT) & -0.043 & 0.610 & 0.033 & 0.822 & -0.008 & 0.956 \\
\hline Insulin (OGTT 0 min) & 0.076 & 0.372 & -0.042 & 0.777 & -0.151 & 0.280 \\
\hline Insulin (OGTT 120 min) & 0.068 & 0.421 & -0.177 & 0.230 & -0.007 & 0.959 \\
\hline C-peptide 0 min (OGTT) & 0.022 & 0.794 & -0.172 & 0.243 & -0.127 & 0.364 \\
\hline C-peptide $120 \min$ (OGTT) & -0.017 & 0.844 & -0.263 & 0.071 & -0.045 & 0.752 \\
\hline Urea nitrogen & 0.102 & 0.227 & 0.258 & 0.076 & 0.282 & 0.041 \\
\hline $\mathrm{Cr}$ & 0.296 & $<0.001$ & 0.469 & 0.001 & 0.423 & 0.002 \\
\hline UA & 0.211 & 0.012 & 0.415 & 0.003 & 0.083 & 0.553 \\
\hline TBIL & -0.010 & 0.902 & 0.213 & 0.146 & 0.142 & 0.310 \\
\hline DBIL & 0.039 & 0.642 & 0.319 & 0.027 & -0.061 & 0.666 \\
\hline IBIL & -0.037 & 0.661 & 0.176 & 0.231 & 0.361 & 0.008 \\
\hline GPT & 0.051 & 0.551 & 0.084 & 0.571 & 0.289 & 0.036 \\
\hline AST & 0.070 & 0.408 & 0.119 & 0.422 & 0.306 & 0.026 \\
\hline ALT & 0.196 & 0.020 & 0.449 & 0.001 & 0.050 & 0.720 \\
\hline ALP & -0.124 & 0.142 & -0.235 & 0.107 & 0.251 & 0.070 \\
\hline LDH & 0.065 & 0.446 & 0.056 & 0.708 & 0.188 & 0.178 \\
\hline TG & -0.088 & 0.297 & 0.059 & 0.689 & 0.135 & 0.334 \\
\hline TC & 0.023 & 0.784 & 0.046 & 0.755 & -0.062 & 0.660 \\
\hline HDL-C & 0.129 & 0.129 & 0.062 & 0.677 & 0.116 & 0.408 \\
\hline LDL-C & 0.035 & 0.680 & -0.227 & 0.121 & 0.215 & 0.122 \\
\hline VLDL-C & 0.345 & $<0.001$ & 0.159 & 0.280 & 0.411 & 0.002 \\
\hline Apo-B & 0.375 & $<0.001$ & 0.256 & 0.079 & -0.030 & 0.829 \\
\hline Apo-A1 & 0.127 & 0.134 & 0.064 & 0.666 & -0.034 & 0.811 \\
\hline HOMA-IR & 0.082 & 0.333 & -0.020 & 0.893 & 0.026 & 0.853 \\
\hline HOMA- $\beta$ & 0.034 & 0.692 & -0.074 & 0.619 & 0.061 & 0.663 \\
\hline ISI & -0.103 & 0.225 & 0.024 & 0.869 & 0.028 & 0.844 \\
\hline
\end{tabular}

Apo-A1 - apolipoprotein-A1, Apo-B - apolipoprotein-B, ALP - alkaline phosphatase, ALT - alanine transaminase, AST - aspartate aminotransferase, $B M I$ - body mass index, $B U$ - blood urea, $\mathrm{Cr}$ - creatine, DBIL - direct bilirubin, DBP - diastolic blood pressure, FBG - fasting blood glucose, GPT - glutamic-pyruvic transaminase, HDL-C - high-density lipoprotein cholesterol, IBIL - indirect bilirubin, $L D H$ - lactate dehydrogenase, LDL-C - low-density lipoprotein cholesterol, NT2DM - newly diagnosed type 2 diabetes mellitus, $N C$ - normal control, RBP-4 - retinol binding protein-4, PDM - prediabetes mellitus, SBP - systolic blood pressure, TC - total cholesterol, $T G$ - triglycerides, TBIL - total bilirubin, UA - uric acid, VLDL-C - very low-density lipoprotein cholesterol, WC - waist circumference, WHR - waist-to-hip ratio. 
A

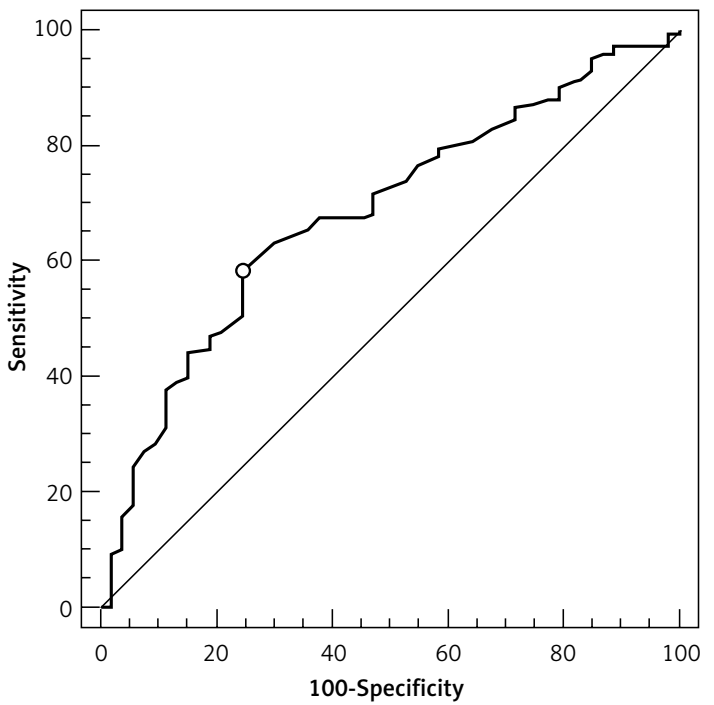

B

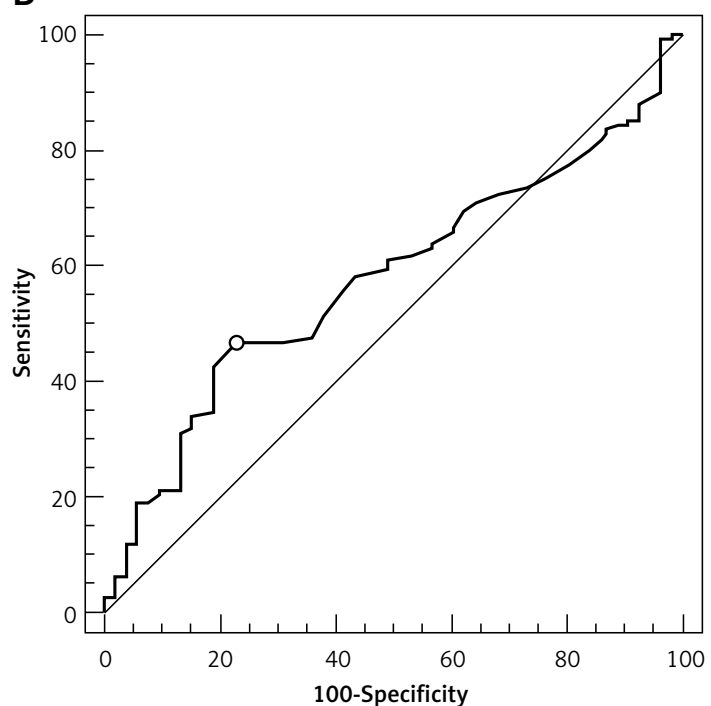

Figure 1. A-Receiver operating characteristic (ROC) curve analysis of Cys C for the discriminative ability of NT2DM patients versus healthy controls. B - Receiver operating characteristic (ROC) curve analysis of hs-CRP for the discriminative ability of NT2DM patients versus healthy controls

A

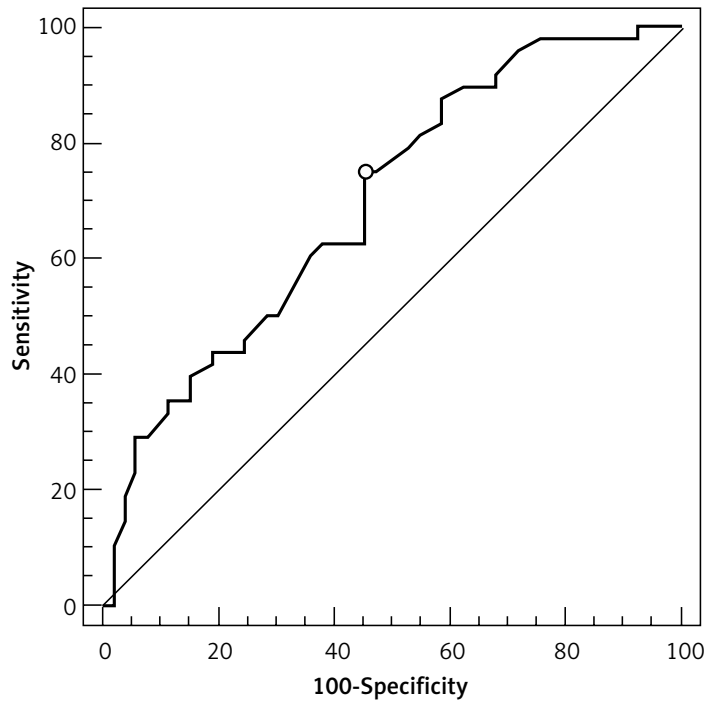

B

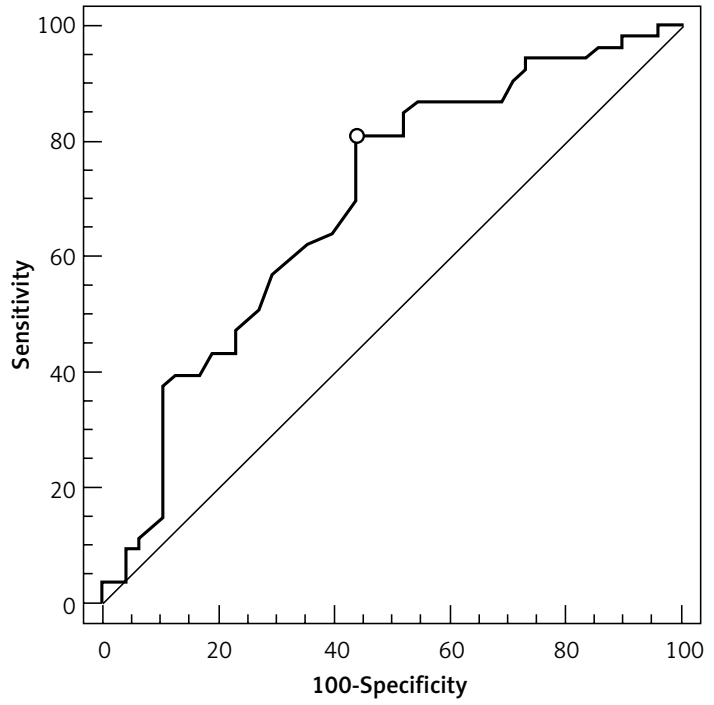

Figure 2. A - Receiver operating characteristic (ROC) curve analysis of Cys C for the discriminative ability of PDM subjects versus healthy controls. B - Receiver operating characteristic (ROC) curve analysis of hs-CRP for the discriminative ability of PDM subjects versus healthy controls

cantly higher hs-CRP level in the PDM group than in normal controls, but we did not observe any significant differences of hs-CRP between NT2DM and normal controls, as well as NT2DM and PDM groups. It is not consistent with previous studies, which suggested that there was an elevated hsCRP concentration in T2DM patients compared to healthy controls, and this elevated level of hs-CRP might be an increased risk of diabetes as well [42, 45-47]. The differences in selection of the study population (NT2DM and PDM subjects in our study versus T2DM patients with or without diabetic complications) may cause this discrepancy in hs-CRP concentration. In addition, it has also been reported that hs-CRP has an association with insulin resistance, of which CRP may contribute to vascular inflammation and cause injury of vascular cells and further contribute to the development of insulin resistance.

Circulating $\mathrm{HCY}$ concentrations have been studied in different populations, and the results are not consistent, including lower, higher or unchanged HCY levels in T2DM patients compared to healthy controls [48-51]. However, a meta-analysis of case-control studies demonstrated that there was a significantly higher pooled HCY level in T2DM patients than in healthy controls [52]. In the present study, we did not find any significant 
differences in NT2DM, PDM and normal controls. Therefore, the relationships between circulating $\mathrm{HCY}$ concentrations and T2DM or PDM remain to be further studied.

Several studies have shown that there was an association of RBP-4 with abnormal glucose tolerance, and an elevated RBP-4 concentration in the T2DM group compared to the healthy control group [13, 17, 53]. In addition, it has been reported that RBP-4 is associated with obesity and insulin resistance. Perseghin et al. found that RBP-4 concentration was associated with peripheral insulin sensitivity and correlated with the soleus intramyocellular lipid (IMCL) content and with the intrahepatic lipid (IHL) content [54]. Shaker et al. reported that there an increased RBP-4 concentration correlated with $\mathrm{BMI}$, waist/hip ratio, insulin and HOMA-IR. Furthermore, RBP-4 correlated with visceral fat and liver fat in diabetic patients [55]. The present analysis did not reveal differences of RBP-4 concentration among NT2DM, PDM and normal control groups, but the correlation analysis revealed an association between RBP-4 and some clinical and laboratory indexes in NT2DM and PDM groups. The difference of RBP-4 concentration may be attributed to the possible ethnic variation. In addition, the measurement methods used in this study might have influenced the RBP-4 level, compared with methods such as quantitative western blotting (WB), and this could lead to the varied results reported [56]. Therefore, further studies are still needed to study ethnic variations of RBP-4 among different populations.

Nevertheless, several other methodological issues and limitations of our study need to be acknowledged. First, the study design was a case-control, observational study, lacking a clear time structure, and a causal relationship between RBP-4, Cys C, HCY and hs-CRP and NT2DM could not be proved. Second, the variations of different ELISA kits when performing detection of the same plasma factor may lead to unstable measurement results. Furthermore, due to a relatively small sample size and single human race, it may restrict the generalizability of our results. Hence, further studies with a large sample size or in multiple human races are still required to confirm our results.

In conclusion, decreased serum Cys $C$ levels and their association with some clinical and laboratory parameters in NT2DM suggest their potential roles in T2DM. In addition, the results of ROC showed relatively high specificity of Cys $C$ and hsCRP for identifying the T2DM high-risk population, indicating useful biomarkers for the differential diagnosis of T2DM. However, further mechanism studies and longitudinal large-cohort studies are still needed to further reveal the role of Cys $C$ and hs-CRP in the pathogenesis of T2DM.

\section{Acknowledgments}

Yun-Sheng Wang and Jun Ye contributed equally to this work and should be considered co-first authors.

The authors thank the study participants as well as the staff involved in the collection of blood samples.

This study was supported by Anhui Science and Technology Project (grant number 1604a0802099).

\section{Conflict of interest}

The authors declare no conflict of interest.

\section{References}

1. Lin Y, Sun Z. Current views on type 2 diabetes. J Endocrinol 2010; 204: 1-11.

2. Lehto S, Ronnemaa T, Haffner SM, Pyorala K, Kallio V, Laakso M. Dyslipidemia and hyperglycemia predict coronary heart disease events in middle-aged patients with NIDDM. Diabetes 1997; 46: 1354-9.

3. Svensson AM, Miftaraj M, Franzen S, Eliasson B. Clinical effects, cardiovascular and renal outcomes associated with rapid-acting insulin analogs among individuals with type 2 diabetes: a nation-wide observational cohort study. Clin Diabetes Endocrinol 2017; 3: 5.

4. Sun X, He J, Ji XL, et al. Association of chronic kidney disease with coronary heart disease and stroke risks in patients with type 2 diabetes mellitus: an observational cross-sectional study in Hangzhou, China. Chin Med J (Engl) 2017; 130: 57-63.

5. Patti AM, Al-Rasadi K, Giglio RV, et al. Natural approaches in metabolic syndrome management. Arch Med Sci 2018; 14: 422-41.

6. Mussap M, Plebani M. Biochemistry and clinical role of human cystatin C. Crit Rev Clin Lab Sci 2004; 41: 467-550.

7. Abrahamson M, Olafsson I, Palsdottir A, et al. Structure and expression of the human cystatin $\mathrm{C}$ gene. Biochem J 1990; 268: 287-94.

8. Gevorgyan MM, Voronina NP, Goncharova NV, et al. Cystatin $\mathrm{C}$ as a marker of progressing cardiovascular events during coronary heart disease. Bull Exp Biol Med 2017; 162: 421-4.

9. Dandana A, Gammoudi I, Chalghoum A, et al. Clinical utility of serum cystatin $C$ in predicting coronary artery disease in patients without chronic kidney disease. J Clin Lab Anal 2014; 28: 191-7.

10. Colantonio LD, Tanner RM, Warnock DG, et al. The role of cystatin- $C$ in the confirmation of reduced glomerular filtration rate among the oldest old. Arch Med Sci 2016; 12: 55-67.

11. Surendar J, Anuradha S, Ashley B, et al. Cystatin C and cystatin glomerular filtration rate as markers of early renal disease in Asian Indian subjects with glucose intolerance (CURES-32). Metab Syndr Relat Disord 2009; 7: 419-25

12. Szopa M, Kapusta M, Matejko B, et al. Comparison of glomerular filtration rate estimation from serum creatinine and cystatin C in HNF1A-MODY and other types of diabetes. J Diabetes Res 2015; 2015: 183094.

13. Takebayashi K, Suetsugu M, Wakabayashi S, Aso Y, Inukai T. Retinol binding protein-4 levels and clinical features of type 2 diabetes patients. J Clin Endocrinol Metab 2007; 92: 2712-9. 
14. Cho YM, Youn BS, Lee $\mathrm{H}$, et al. Plasma retinol-binding protein-4 concentrations are elevated in human subjects with impaired glucose tolerance and type 2 diabetes. Diabetes Care 2006; 29: 2457-61.

15. Kloting N, Graham TE, Berndt J, et al. Serum retinol-bind ing protein is more highly expressed in visceral than in subcutaneous adipose tissue and is a marker of in tra-abdominal fat mass. Cell Metab 2007; 6: 79-87.

16. Jia $W, W u H, B a o ~ Y$, et al. Association of serum retinol-binding protein 4 and visceral adiposity in Chinese subjects with and without type 2 diabetes. J Clin Endocrinol Metab 2007; 92: 3224-9.

17. Wu H, Jia W, Bao Y, et al. Serum retinol binding protein 4 and nonalcoholic fatty liver disease in patients with type 2 diabetes mellitus. Diabetes Res Clin Pract 2008; 79: 185-90.

18. Stipanuk MH. Sulfur amino acid metabolism: pathways for production and removal of homocysteine and cysteine. Annu Rev Nutr 2004; 24: 539-77.

19. Marti-Carvajal AJ, Sola I, Lathyris D. Homocysteine-low ering interventions for preventing cardiovascular events. Cochrane Database Syst Rev 2015; 1: CD006612.

20. Haan MN, Miller JW, Aiello AE, et al. Homocysteine, B vitamins, and the incidence of dementia and cognitive impairment: results from the Sacramento Area Latino Study on Aging. Am J Clin Nutr 2007; 85: 511-7.

21. Wollesen F, Brattstrom L, Refsum H, Ueland PM, Berglund L, Berne C. Plasma total homocysteine and cys teine in relation to glomerular filtration rate in diabetes mellitus. Kidney Int 1999; 55: 1028-35.

22. Katsiki N, Perez-Martinez P, Mikhailidis DP. Homocysteine and non-cardiac vascular disease. Curr Pharm Des 2017; 23: 3224-32

23. Li Y, Jiang C, Xu G, et al. Homocysteine upregulates resistin production from adipocytes in vivo and in vitro. Diabetes 2008; 57: 817-27.

24. Ridker PM. Clinical application of C-reactive protein for cardiovascular disease detection and prevention. Circu lation 2003; 107: 363-9.

25. Kim JW, Han JE, Kim YS, Won HJ, Yoon TK, Lee WS. High sensitivity C-reactive protein and its relationship with impaired glucose regulation in lean patients with polycystic ovary syndrome. Gynecol Endocrinol 2012; 28: 259-63.

26. Yang SP, Gong CX, Cao BY, Yan C. Relationship between serum high-sensitivity $\mathrm{C}$-reactive protein and obesity and impaired glycose metabolism in children and adolescents. Zhonghua Er Ke Za Zhi 2006; 44: 933-6.

27. Brunner EJ, Kivimaki M, Witte DR, et al. Inflammation, insulin resistance, and diabetes--Mendelian randomization using CRP haplotypes points upstream. PLoS Med 2008; 5: e155.

28. Bahceci M, Tuzcu A, Ogun C, Canoruc N, Iltimur K, Aslan C. Is serum $\mathrm{C}$-reactive protein concentration correlated with $\mathrm{HbA1c}$ and insulin resistance in type 2 diabetic men with or without coronary heart disease? J Endocrinol Invest 2005; 28: 145-50.

29. Yuan G, Zhou L, Tang J, et al. Serum CRP levels are equally elevated in newly diagnosed type 2 diabetes and impaired glucose tolerance and related to adiponectin levels and insulin sensitivity. Diabetes Res Clin Pract 2006; 72: 244-50

30. American Diabetes A. 2. Classification and Diagnosis of Diabetes: Standards of Medical Care in Diabetes-2018. Diabetes Care 2018; 41: S13-27.

31. Lansang MC, Williams GH, Carroll JS. Correlation between the glucose clamp technique and the homeosta- sis model assessment in hypertension. Am J Hypertens 2001; 14: 51-3.

32. Takai T, Sakura H. Insulinogenic index, HOMA-beta, disposition index. Nihon Rinsho 2012; 70 Suppl 3: 459-64.

33. Matsuda M, DeFronzo RA. Insulin sensitivity indices obtained from oral glucose tolerance testing: comparison with the euglycemic insulin clamp. Diabetes Care 1999; 22: 1462-70.

34. Chong YB, Keng TC, Tan LP, et al. Clinical predictors of non-diabetic renal disease and role of renal biopsy in diabetic patients with renal involvement: a single centre review. Ren Fail 2012; 34: 323-8.

35. Li L, Zhang X, Li Z, et al. Renal pathological implications in type 2 diabetes mellitus patients with renal involvement. J Diabetes Complications 2017; 31: 114-21.

36. Kanakatti Shankar R, Dolan LM, Isom S, et al. Serum cystatin $\mathrm{C}$ in youth with diabetes: the SEARCH for diabetes in youth study. Diabetes Res Clin Pract 2017; 130: 258-65.

37. Wang T, Wang Q, Wang Z, Xiao Z, Liu L. Diagnostic value of the combined measurement of serum hcy, serum cys $C$, and urinary microalbumin in type 2 diabetes mellitus with early complicating diabetic nephropathy. ISRN Endocrinol 2013; 2013: 407452.

38. Dhia RB, Hellara I, Harzallah O, et al. Evaluation of the renal function in type 2 diabetes: clearance calculation or cystatin C? Ann Biol Clin (Paris) 2012; 70: 287-94.

39. Liu S, Ren J, Xia Q, et al. Preliminary case-control study to evaluate diagnostic values of $\mathrm{C}$-reactive protein and erythrocyte sedimentation rate in differentiating active Crohn's disease from intestinal lymphoma, intestinal tuberculosis and Behcet's syndrome. Am J Med Sci 2013; 346: 467-72.

40. Akpek M, Kaya MG, Lam YY, et al. Relation of neutrophil/ lymphocyte ratio to coronary flow to in-hospital major adverse cardiac events in patients with ST-elevated myocardial infarction undergoing primary coronary intervention. Am J Cardiol 2012; 110: 621-7.

41. Baumann S, Huseynov A, Koepp J, et al. Comparison of serum uric acid, bilirubin, and C-reactive protein as prognostic biomarkers of in-hospital MACE between women and men with ST-segment elevation myocardial infarction. Angiology 2016; 67: 272-80.

42. Pleskovic A, Letonja MS, Vujkovac AC, et al. C-reactive protein as a marker of progression of carotid atherosclerosis in subjects with type 2 diabetes mellitus. Vasa 2017; 46: 187-92.

43. Ribeiro DR, Ramos AM, Vieira PL, et al. High-sensitivity C-reactive protein as a predictor of cardiovascular events after ST-elevation myocardial infarction. Arq Bras Cardiol 2014; 103: 69-75.

44. Aryan Z, Ghajar A, Faghihi-Kashani S, Afarideh M, Nakhjavani M, Esteghamati A. Baseline high-sensitivity C-reactive protein predicts macrovascular and microvascular complications of type 2 diabetes: a population-based study. Ann Nutr Metab 2018; 72: 287-95.

45. Zang H, Jiang F, Cheng X, Xu H, Hu X. Serum adropin levels are decreased in Chinese type 2 diabetic patients and negatively correlated with body mass index. Endocr J 2018; 65: 685-91.

46. Ebtehaj S, Gruppen EG, Parvizi M, Tietge UJF, Dullaart RPF. The anti-inflammatory function of HDL is impaired in type 2 diabetes: role of hyperglycemia, paraoxonase- 1 and low grade inflammation. Cardiovasc Diabetol 2017; 16: 132.

47. Hosny SS, Bahaaeldin AM, Khater MS, Bekhet MM, Hebah HA, Hasanin GA. Role of inflammatory markers in elderly type 2 diabetic patients with mild cognitive im- 
pairment. Curr Diabetes Rev 2018; doi: 10.2174/15733 99814666180423113341.

48. Kundi H, Kiziltunc E, Ates I, et al. Association between plasma homocysteine levels and end-organ damage in newly diagnosed type 2 diabetes mellitus patients. Endocr Res 2017; 42: 36-41.

49. Mao S, Xiang W, Huang S, Zhang A. Association between homocysteine status and the risk of nephropathy in type 2 diabetes mellitus. Clin Chim Acta 2014; 431: 206-10.

50. Ala OA, Akintunde AA, Ikem RT, Kolawole BA, Ala OO, Adedeji TA. Association between insulin resistance and total plasma homocysteine levels in type 2 diabetes mellitus patients in south west Nigeria. Diabetes Metab Syndr 2017; 11 Suppl 2: S803-S9.

51. Upadhyay TR, Kothari N, Shah H. Association between serum B12 and serum homocysteine levels in diabetic patients on metformin. J Clin Diagn Res 2016; 10: BC01-4.

52. Huang T, Ren J, Huang J, Li D. Association of homocysteine with type 2 diabetes: a meta-analysis implementing Mendelian randomization approach. BMC Genomics 2013; 14: 867

53. Liu G, Ding M, Chiuve SE, et al. Plasma levels of fatty acid-binding protein 4 , retinol-binding protein 4 , high-molecular-weight adiponectin, and cardiovascular mortality among men with type 2 diabetes: a 22-year prospective study. Arterioscler Thromb Vasc Biol 2016; 36: 2259-67.

54. Perseghin G, Lattuada G, De Cobelli F, et al. Serum retinol-binding protein-4, leptin, and adiponectin concentrations are related to ectopic fat accumulation. J Clin Endocrinol Metab 2007; 92: 4883-8.

55. Shaker O, El-Shehaby A, Zakaria A, et al. Plasma visfatin and retinol binding protein-4 levels in patients with type 2 diabetes mellitus and their relationship to adiposity and fatty liver. Clin Biochem 2011; 44: 1457-63.

56. Graham TE, Wason CJ, Bluher M, Kahn BB. Shortcomings in methodology complicate measurements of serum retinol binding protein (RBP4) in insulin-resistant human subjects. Diabetologia 2007; 50: 814-23. 